JOURNAL OF SYNCHROTRON RADIATION

ISSN 1600-5775

Received 11 October 2019

Accepted 7 January 2020

Edited by S. M. Heald, Argonne National Laboratory, USA

Keywords: transmission X-ray microscopy; deep learning; limited angle tomography.
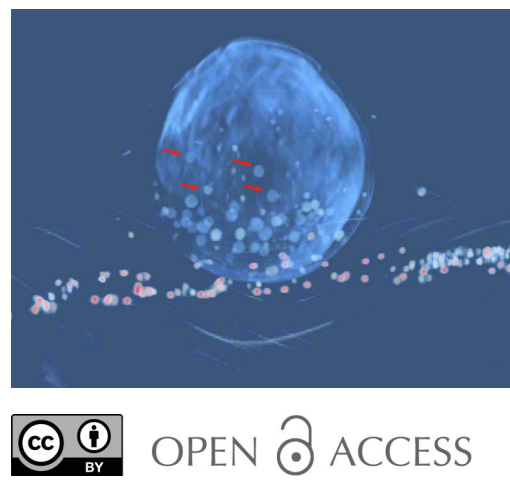

\section{Limited angle tomography for transmission X-ray microscopy using deep learning}

\author{
Yixing Huang, ${ }^{a} *$ Shengxiang Wang,,${ }^{b, c} *$ Yong Guan ${ }^{\mathrm{d}}$ and Andreas Maier ${ }^{\mathrm{a}, \mathrm{e}}$ \\ aPattern Recognition Lab, Friedrich-Alexander-Universität Erlangen-Nürnberg, 91058 Erlangen, Germany, \\ ${ }^{\text {b}}$ Spallation Neutron Source Science Center, Dongguan, Guangdong 523803, People's Republic of China, \\ 'Institute of High Energy Physics, Chinese Academy of Sciences, Beijing 100049, People's Republic of China, \\ dNational Synchrotron Radiation Laboratory, University of Science and Technology of China, Hefei, \\ Anhui 230026, People's Republic of China, and 'Erlangen Graduate School in Advanced Optical Technologies \\ (SAOT), 91052 Erlangen, Germany. *Correspondence e-mail: yixing.yh.huang@fau.de, wangsx@ihep.ac.cn
}

In transmission X-ray microscopy (TXM) systems, the rotation of a scanned sample might be restricted to a limited angular range to avoid collision with other system parts or high attenuation at certain tilting angles. Image reconstruction from such limited angle data suffers from artifacts because of missing data. In this work, deep learning is applied to limited angle reconstruction in TXMs for the first time. With the challenge to obtain sufficient real data for training, training a deep neural network from synthetic data is investigated. In particular, U-Net, the state-of-the-art neural network in biomedical imaging, is trained from synthetic ellipsoid data and multi-category data to reduce artifacts in filtered back-projection (FBP) reconstruction images. The proposed method is evaluated on synthetic data and real scanned chlorella data in $100^{\circ}$ limited angle tomography. For synthetic test data, U-Net significantly reduces the root-mean-square error (RMSE) from $2.55 \times 10^{-3} \mu \mathrm{m}^{-1}$ in the FBP reconstruction to $1.21 \times 10^{-3} \mu \mathrm{m}^{-1}$ in the U-Net reconstruction and also improves the structural similarity (SSIM) index from 0.625 to 0.920 . With penalized weighted least-square denoising of measured projections, the RMSE and SSIM are further improved to $1.16 \times 10^{-3} \mu \mathrm{m}^{-1}$ and 0.932 , respectively. For real test data, the proposed method remarkably improves the $3 \mathrm{D}$ visualization of the subcellular structures in the chlorella cell, which indicates its important value for nanoscale imaging in biology, nanoscience and materials science.

\section{Introduction}

Transmission X-ray microscopy (TXM) has become a very powerful technology for nanoscale imaging in various fields (Wang et al., 2000, 2016; Chao et al., 2005; Sakdinawat \& Attwood, 2010), including materials science (Andrews et al., 2011; Nelson et al., 2012), chemistry (de Smit et al., 2008; Wang et al., 2015a) and biology (Shapiro et al., 2005; Wang et al., $2015 b)$. With projection images acquired in a series of rotational angles, tomographic images can be reconstructed according to computed tomography (CT) technologies for 3D visualization of scanned samples. In such applications, TXM is also called X-ray nano-CT (Shearing et al., 2011; Brisard et al., 2012; Liu et al., 2018). A TXM system typically consists of a central stop, a condenser, a sample holder, an objective zone plate and a CCD detector, with X-rays generated from synchrotron radiation or a high-end X-ray source. TXMs typically utilize a pin as the sample holder (Holler et al., 2017), e.g. tip versions for pillar samples, glass capillaries for powder samples, copper capillaries for high-pressure cryogenic samples and grids for flat samples. For tips and capillaries, 
rotating a sample in a sufficient angular range is not a problem. However, for grids, collision between the grid and the zone plate, which is very near to the rotation axis in TXM systems, might happen at large scan angles. In addition, for flat samples, the lengths of X-rays through the sample increase rapidly at high tilting angles (Barnard et al., 1992; Koster et al., 1997), which introduces a high level of scattering and reduces image contrast. Therefore, in these situations, the problem of limited angle tomography arises.

Limited angle tomography is a severely ill-posed inverse problem (Davison, 1983; Louis, 1986; Natterer, 1986; Quinto, 2006). Using microlocal analysis, edges that are tangent to available X-rays can be well reconstructed while those whose singularities are not perpendicular to any X-ray lines cannot be reconstructed stably (Quinto, 1993, 2006). So far, many algorithms have been developed to deal with this task. Among these algorithms, extrapolating missing data is the most straightforward way for limited angle tomography. The iterative Gerchberg-Papoulis extrapolation algorithm (Gerchberg, 1974; Papoulis, 1975) based on band-limitation properties of imaged objects has been demonstrated beneficial for improving image quality of limited angle tomography (Defrise \& de Mol, 1983; Qu et al., 2008; Qu \& Jiang, 2009; Huang et $a l ., 2018 b$ ). In addition, data-consistency conditions, e.g. the Helgason-Ludwig consistency conditions (Helgason, 1965; Ludwig, 1966), provide redundancy and constraint information of projection data, which effectively improves the quality of extrapolation (Louis \& Törnig, 1980; Louis, 1981; Prince \& Willsky, 1990; Kudo \& Saito, 1991; Huang et al., 2017). Nevertheless, such extrapolation methods have only achieved limited performance on real data, which typically contain complex structures and are very difficult to extrapolate.

Iterative reconstruction using sparse regularization technologies, particularly total variation (TV), has been widely applied to image reconstruction from insufficient data. TV methods employ the sparsity information of image gradients as a regularization term. Therefore, noise and artifacts, which tend to increase the TV value, can be reduced via such regularization. For limited angle tomography, algorithms of adaptive steepest descent projection onto convex sets (ASDPOCS) (Sidky et al., 2006; Sidky \& Pan, 2008), improved total variation (iTV) (Ritschl et al., 2011), anisotropic total variation (aTV) (Chen et al., 2013), reweighted total variation (wTV) (Huang et al., 2016a, 2016b) and scale-space anisotropic total variation (ssaTV) (Huang et al., 2018a) have been proposed. While TV methods achieve good reconstruction results when the missing angular range is small, they fail to reduce severe artifacts when a large angular range is missing. Moreover, they also require expensive computation and tend to lose high-resolution details.

Recently, machine-learning techniques have achieved overwhelming success in a large range of fields including X-ray imaging. In the application of limited angle tomography, pixelby-pixel artifact prediction using traditional machine learning is one direction (Huang et al., 2019a). However, new artifacts might be introduced. Instead, deep-learning methods have achieved impressive results. Würfl et al. $(2016,2018)$ proposed to learn certain weights based on known filtered backprojection (FBP) operators (Maier et al., 2019) to compensate missing data in limited angle tomography. Gu \& Ye (2017) proposed to learn artifacts from streaky images in a multiscale wavelet domain using the U-Net architecture (Ronneberger et al., 2015; Falk et al., 2019). Bubba et al. (2019) utilized an iterative shearlet transform algorithm to reconstruct visible singularities of an imaged object and a U-Net based neural network with dense blocks to predict invisible singularities. In our previous work, we have demonstrated that deep learning is not robust to noise and adversarial examples (Huang et al., 2018c). To improve image quality, a data consistent reconstruction method (Huang et al., 2019b) is proposed, where deep-learning reconstruction is used as prior to provide information of missing data while conventional iterative reconstruction is applied to make deep-learning reconstruction consistent to measured projection data.

In this work, deep learning is applied to limited angle reconstruction in the field of TXMs for the first time, to the best of our knowledge. Furthermore, training data is vital for deep-learning methods. Without access to real training data, in this work we will investigate the performance of deep learning trained from synthetic data.

\section{Materials and method}

The proposed limited angle reconstruction method for TXMs consists of two steps: FBP preliminary reconstruction and deep-learning reconstruction as post-processing.

\subsection{FBP preliminary reconstruction}

For TXM systems with synchrotron radiation, parallel-beam $\mathrm{X}$-rays are used. Each X-ray measures a line integral of the linear attenuation coefficients of a scanned sample, represented as

$$
\begin{aligned}
\boldsymbol{p}(u, v, \theta)= & \iiint_{-\infty}^{\infty} \boldsymbol{f}(x, y, z) \\
& \times \delta(x \cos \theta+y \sin \theta-u, z-v) \mathrm{d} x \mathrm{~d} y \mathrm{~d} z
\end{aligned}
$$

where $\theta$ is the rotation angle of the sample, the rotation axis is parallel with the $z$ axis, $u$ and $v$ are the horizontal and vertical position indices at the detector, respectively, $\boldsymbol{p}(u, v, \theta)$ is the log-transformed projection, $\boldsymbol{f}(x, y, z)$ is the attenuation distribution function of the sample, and $\delta(\cdot)$ is the Dirac delta function.

In practice, noise always exists in measured projections because of various physical effects, e.g. Poisson noise. Since deep-learning methods are sensitive to noise (Huang et al., $2018 c$ ), noise reduction in input images is preferred. For this purpose, a penalized weighted least-square (PWLS) approach is utilized in projection domain. The objective function for PWLS is as follows (Wang et al., 2006),

$$
\Phi(\boldsymbol{p})=(\hat{\boldsymbol{p}}-\boldsymbol{p})^{\top} \Lambda^{-1}(\hat{\boldsymbol{p}}-\boldsymbol{p})+\beta \cdot R(\boldsymbol{p}),
$$


where $\boldsymbol{p}$ is the vector of the ideal log-transformed projection, $\hat{\boldsymbol{p}}$ is the vector of the measured log-transformed projection containing noise, $\boldsymbol{p}_{i}$ is the $i$ th element of $\boldsymbol{p}, \Lambda$ is a diagonal matrix with the $i$ th element equal to an estimate of the variance of $\hat{\boldsymbol{p}}_{i}, R(\boldsymbol{p})$ is a regularization term and $\beta$ is a relaxation parameter. The regularization term $R(\boldsymbol{p})$ is chosen as

$$
R(\boldsymbol{p})=\frac{1}{2} \sum_{i} \sum_{j \in \mathcal{N}_{i}} w_{i, j}\left(\boldsymbol{p}_{i}-\boldsymbol{p}_{j}\right)^{2},
$$

where $\mathcal{N}_{i}$ is the four-connectivity neighborhood of the $i$ th pixel and the weight $w_{i, j}$ is defined as

$$
w_{i, j}=\exp \left[-\left(\boldsymbol{p}_{i}-\boldsymbol{p}_{j}\right)^{2} / \sigma^{2}\right]
$$

with $\sigma$ a predefined parameter to control the weight.

The denoised projection is denoted by $\boldsymbol{p}^{\prime}(u, v, \theta)$. For image reconstruction, the FBP algorithm with the Ram-Lak kernel $\boldsymbol{h}(u)$ is applied,

$$
\begin{aligned}
\left.\boldsymbol{f}_{\mathrm{FBP}, \mathrm{PWLS}}(x, y, z)\right|_{z=v}= & \int_{\theta_{\min }}^{\theta_{\max }} \int_{-\infty}^{\infty} \boldsymbol{p}^{\prime}(u, v, \theta) \\
& \times \boldsymbol{h}(x \cos \theta+y \sin \theta-u) \mathrm{d} u \mathrm{~d} \theta,
\end{aligned}
$$

where $\theta_{\min }$ and $\theta_{\max }$ are the start rotation angle and the end rotation angle, respectively, and $\boldsymbol{f}_{\mathrm{FBP}}$, PWLS is the FBP reconstruction from PWLS processed projection data. We further denote the FBP reconstruction from measured projection data without PWLS by $\boldsymbol{f}_{\mathrm{FBP}}$, i.e. replacing $\boldsymbol{p}^{\prime}(u, v, \theta)$ by $\hat{\boldsymbol{p}}(u, v, \theta)$ in the above equation.

\subsection{Deep-learning reconstruction}

2.2.1. Neural network. The above FBP reconstruction suffers from artifacts, typically in the form of streaks, because of missing data in limited angle tomography. To reduce artifacts, an image-to-image post-processing deep-learning method using U-Net is applied.

The U-Net architecture for limited angle tomography is displayed in Fig. 1. The input and output of the U-Net are both 2D images of the same size. Each blue arrow stands for zeropadded $3 \times 3$ convolution followed by a rectified linear unit ( $\mathrm{ReLu}$ ), a batch normalization (BN) operation, and a squeezeand-extraction (SE) block (Hu et al., 2018). Each red arrow represents a max pooling operation to down-sample feature maps by a factor of two. Each green arrow is a bilinear upsampling operation followed by a $2 \times 2$ convolution to resize

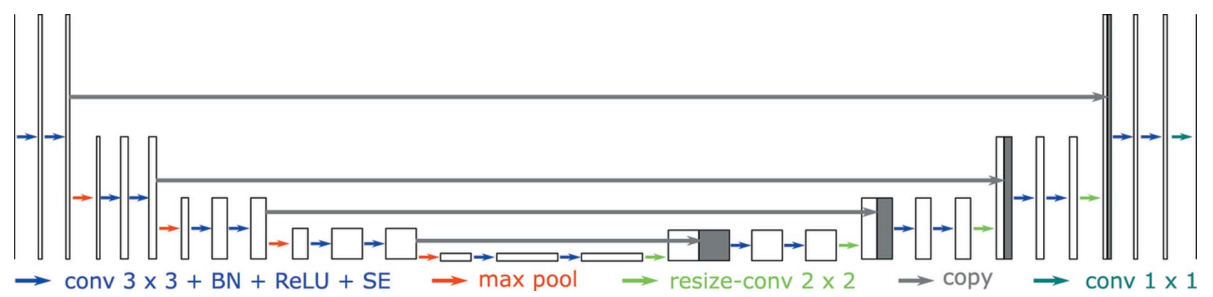

Figure 1

The U-Net architecture for limited angle tomography. feature maps back. The gray arrows copy features from the left side and concatenate them with the corresponding up-sampled features. The last $1 \times 1$ convolution operation maps the multichannel features to a desired output image. Because of the down/up-sampling and copy operations, the U-Net architecture has a large reception field and is able to learn features of multi-scales.

In this work, the input image is a $2 \mathrm{D}$ horizontal slice from the FBP reconstruction without or with PWLS pre-processing, i.e. $f_{\mathrm{FBP}}$ or $\boldsymbol{f}_{\mathrm{FBP}}$, PWLS , respectively. The output image is the corresponding artifact image. Hence, a final reconstruction of the U-Net, denoted by $\boldsymbol{f}_{\mathrm{U}-\mathrm{Net}}$ or $\boldsymbol{f}_{\mathrm{U}-\mathrm{Net} \text {, PWLS }}$ for the input image without and with PWLS, respectively, is obtained by subtracting the input image by its corresponding predicted artifact image. For stable training, the input and output images are normalized to the range $[-1,1]$ using the maximum intensity value of the input images.

Compared with the original U-Net architecture in the work by Ronneberger et al. (2015), the following modifications are made in the above U-Net architecture to improve its performance for limited angle tomography.

(i) Zero-padded convolution. In the original U-Net architecture, unpadded convolution is used and the image size decreases after each convolution. Hence, information near image boundaries is missing in the output image. In this work, the zero-padded convolution is used to preserve image size. Because of this, the cropping operation is no longer necessary for each copy operation.

(ii) Batch normalization. The $\mathrm{BN}$ operation normalizes each convolutional layer's inputs in a mini-batch to a normal distribution with trained mean shift and variance-scaling values. The $\mathrm{BN}$ technique allows neural networks to use higher learning rates and be less sensitive to initialization (Ioffe \& Szegedy, 2015). Therefore, it is a standard operation for convolutional neural networks nowadays.

(iii) Squeeze-and-extraction. The SE block (Hu et al., 2018) squeezes global spatial information into a channel descriptor by first using global average pooling. Afterwards, channel-wise dependencies are captured by a nonlinear excitation mechanism, which emphasizes multi-channel activations instead of single-channel activation. The SE technique adaptively recalibrates channel-wise feature responses to boost the representation power of a neural network.

(iv) Resize and $2 \times 2$ convolution. The original U-Net architecture uses a deconvolution operation for up-sampling, which introduces checkerboard artifacts (Odena et al., 2016). To avoid this, we first choose to resize each feature map using bilinear up-sampling with a scaling factor of two. Afterwards, a $2 \times 2$ convolution operation is applied.

(v) Output and loss function. The original U-Net is proposed for biomedical image segmentation, where the number of segmentation classes decides the channel number of the output image and each channel is a binary vector containing elements of 
0 or 1 . For segmentation, a softmax function is typically used to determine the highest probability class. Associated with the softmax activation in the output layer, the cross entropy loss function is typically used for training. As mentioned previously, the output image is a one-channel $2 \mathrm{D}$ artifact image in this work. Therefore, the result of the $1 \times 1$ convolution is directly used as the output without any softmax function. Correspondingly, an $\ell_{2}$ loss function is used for training.

2.2.2. Data preparation. In order to reconstruct a sample from limited angle data using deep learning, training data is vital. However, on the one hand it is very challenging to obtain a sufficient amount of real data; on the other hand, for most scans only limited angle data are acquired and hence reconstruction from complete data as ground truth is not available. Because of the scarcity of real data, we choose to train the neural network from synthetic data. For this purpose, two kinds of synthetic data are generated.

(i) Ellipsoid phantoms. 3D ellipsoid phantoms are designed with two large ellipsoids to form an outer boundary, two middle-sized ellipsoids to simulate the cup-shaped chloroplast, 20 small ellipsoids to mimic lipid bodies, and 50 high-intensity small-sized ellipsoids to simulate gold nanoparticles which are contained in the sample for geometry and motion calibration (Wang et al., 2019). The locations, sizes and intensities of the ellipsoids are randomly generated. Since many samples are immobilized in a certain condition, e.g. in an ice tube in this work, a background with a constant intensity of $0.002 \mu \mathrm{m}^{-1}$ is added.

(ii) Multi-category data. For a certain parallel-beam limited angle tomography system, no matter what kinds of objects are imaged, the projections and the FBP reconstructions should follow the mathematics in equations (1) and (5). In addition, based on the theories of transfer learning (Pan \& Yang, 2010) and one/zero-shot learning ( $\mathrm{Li}$ et al., 2006; Palatucci et al., 2009), a neural network trained for one task can also generalize to another similar task. Therefore, in this work, images of multi-categories are collected to train the neural network for complex structures, for example, optical microscopy algae images and medical CT images. Note that although TXMs data for chlorella cells, the test sample in this work, are not accessible, data of algae cells in other imaging modalities, especially in optical microscopies, are abundant. Images in other modalities also share plenty of useful structure information as TXMs do.

\subsection{Experimental setup}

2.3.1. Synthetic data. For deep-learning training, 10 ellipsoid phantoms with a size of $512 \times 512 \times 512$ are generated. From each 3D phantom, 20 slices are uniformly selected. From the multi-category data, 400 image slices are collected. Color images are converted to gray intensity images. The above images are further rotated by 90,180 and $270^{\circ}$. Therefore, 2400 image slices in total are synthesized for training.

Parallel-beam sinograms are simulated from rotation angles $-50^{\circ}$ to $50^{\circ}$ with an angular step of $1^{\circ}$, as displayed in Fig. 2 .

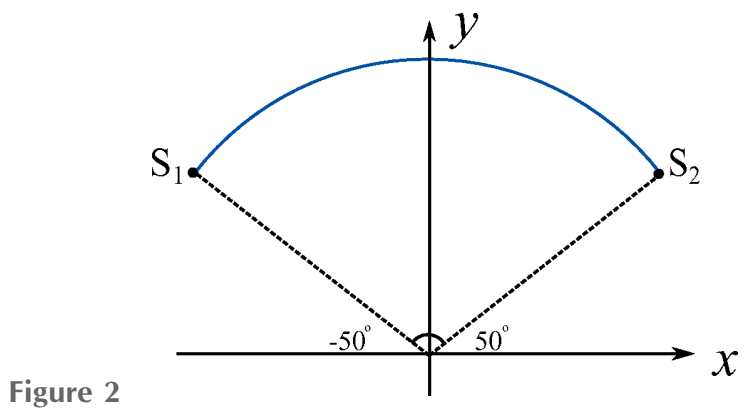

The scanned angular range of the TXM system is from $-50^{\circ}$ to $50^{\circ}$.

The detector size is 512 with a pixel size of $21.9 \mathrm{~nm}$. To improve the robustness of the neural network to noise, Poisson noise is simulated considering a photon number of $10^{4}, 5.0 \times 10^{4}$ or $10^{5}$ for each X-ray before attenuation. Here, multiple dose levels are used to improve the robustness of the neural network to different levels of noise. For training, 1200 preliminary image slices with a size of $256 \times 256$ are reconstructed by FBP using the Ram-Lak kernel directly from noisy projection data for the 600 original slices and their $90^{\circ}$ rotations, while the other 1200 slices are reconstructed from projection data processed by two iterations of PWLS. To obtain the diagonal matrix $\Lambda$ in equation (2), the variance of each detector pixel $\hat{\boldsymbol{p}}_{i}$ is estimated by the following formula (Wang et al., 2006),

$$
\sigma_{i}^{2}=a_{i} \cdot \exp \left(\hat{\boldsymbol{p}}_{i} / \eta\right)
$$

where $a_{i}$ is set to 0.5 for each pixel $i$ and $\eta$ is set to 1 . The value of $\sigma$ in equation (4) is set to 2 .

The U-Net is trained on the above synthetic data using the Adam optimizer for 500 epochs. The learning rate is $10^{-3}$ for the first 100 epochs and gradually decreases to $10^{-5}$ for the final epochs. The $\ell_{2}$-regularization with a parameter of $10^{-4}$ is applied to avoid large network weights.

For a preliminary quantitative evaluation, the trained U-Net model is evaluated on one new synthetic ellipsoid phantom first. Its limited angle projection data are generated with Poisson noise using a photon number of $10^{4}$. The projections are denoised by two iterations of PWLS.

2.3.2. Chlorella data. As a demonstration example, a sample of chlorella cells was scanned in a soft X-ray microscope at beamline BL07W (Liu et al., 2018) in the National Synchrotron Radiation Laboratory in Hefei, China. Chlorella is a genus of single-celled green algae with a size of 2 to $10 \mu \mathrm{m}$. It mainly consists of a single- to triple-layered cell wall, a thin plasma membrane, a nucleus, a cup-shaped chloroplast, a pyrenoid and several lipid bodies, as illustrated in Fig. 3 (Baudelet et al., 2017).

To hold the chlorella sample, a traditional 100 mesh transmission electron microscopy (TEM) grid was used. Because of the TEM grid, a valid scan of $100^{\circ}$ only $\left(-50^{\circ}\right.$ to $50^{\circ}$ in Fig. 2 with an angular step of $1^{\circ}$ ) was acquired to avoid collision between the grid and the zone plate. Rapid freezing of the chlorella sample with liquid nitrogen was performed before scanning to immobilize the cells in an ice tube and suppress the damage of radiation to cellular structures. The X-ray 


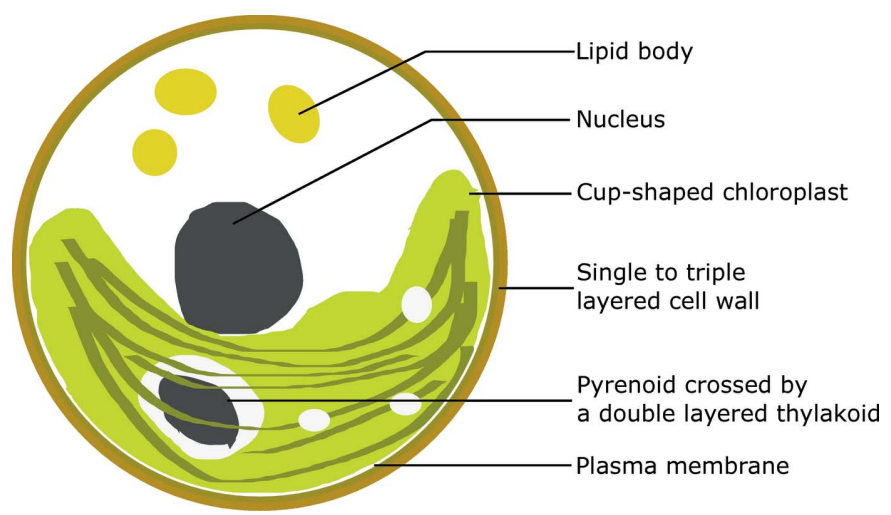

Figure 3

The main structures of a chlorella cell (Baudelet et al., 2017).

energy used in the experiment was $520 \mathrm{eV}$ for the so-called 'water window'. Each projection image is rebinned to a size of $512 \times 512$ with a pixel size of $21.9 \mathrm{~nm} \times 21.9 \mathrm{~nm}$. As the shift of rotation axis (Yang et al., 2015) and jitter motion (Yu et al., 2018) are two main causes of image blurriness, they were solved via measurement of geometric moments after acquisition, as described in the work by Wang et al. (2019). The projections are denoised by two iterations of PWLS afterwards.

\section{Results and discussion}

\subsection{Ellipsoid phantom results}

The reconstruction results without and with PWLS for the 250th slice of the test ellipsoid phantom using a photon number of $10^{4}$ are displayed in Fig. 4. The root-mean-square error (RMSE) inside the field-of-view (FOV) of each image slice with respect to the corresponding reference slice is displayed in the subcaption. In Figs. 4(b)-4(e), the outer ring is caused by the lateral truncation and it is preserved to mark the FOV.

The FBP reconstruction from $100^{\circ}$ limited angle data without PWLS pre-processing, $\boldsymbol{f}_{\mathrm{FBP}}$, is displayed in Fig. 4(b). Compared with the reference image $f_{\text {Reference }}$, only the structures with an orientation inside the scanned angular range (Fig. 2) are reconstructed while all other structures are severely distorted. In addition, the Poisson noise pattern is clearly observed because of the low dose. In contrast, Poisson noise is prominently reduced by PWLS in $\boldsymbol{f}_{\mathrm{FBP}, \text { PWLS }}$, as displayed in Fig. 4(c). The U-Net reconstruction with the input of $\boldsymbol{f}_{\mathrm{FBP}}$ is displayed in Fig. 4(d), where most ellipsoid boundaries are restored well.

Figure 4
Table 1

The average RMSE and SSIM values for each reconstruction method using a photon number of $10^{4}$ without or with PWLS.

\begin{tabular}{lllll}
\hline Metric & $\boldsymbol{f}_{\mathrm{FBP}}$ & $\boldsymbol{f}_{\mathrm{FBP}, \mathrm{PWLS}}$ & $\boldsymbol{f}_{\mathrm{U}-\mathrm{Net}}$ & $\boldsymbol{f}_{\mathrm{U}-\mathrm{Net}, \mathrm{PWLS}}$ \\
\hline RMSE $\left(10^{-3} \mu \mathrm{m}^{-1}\right)$ & 2.55 & 2.44 & 1.21 & 1.16 \\
SSIM & 0.625 & 0.648 & 0.920 & 0.932 \\
\hline
\end{tabular}

The RMSE inside the FOV is reduced from $3.61 \times 10^{-3} \mu \mathrm{m}^{-1}$ in $\boldsymbol{f}_{\mathrm{FBP}}$ to $1.65 \times 10^{-3} \mu \mathrm{m}^{-1}$ in $\boldsymbol{f}_{\mathrm{U}-\mathrm{Net}}$. This demonstrates the efficacy of deep learning in artifact reduction for limited angle tomography. However, some Poisson noise remains in Fig. $4(d)$. In particular, the boundary indicated by the red arrow is disconnected in $f_{\mathrm{U}-\mathrm{Net}}$. The U-Net reconstruction with the input of $\boldsymbol{f}_{\mathrm{FBP}, \text { PWLS }}$ is displayed in Fig. 4(e), achieving the smallest RMSE value of $1.58 \times 10^{-3} \mu^{-1}$. Importantly, the disconnected boundary fragment indicated by the red arrow is reconstructed in $\boldsymbol{f}_{\mathrm{U}-\mathrm{Net} \text {, PWLS }}$. This demonstrates the benefit of PWLS pre-processing.

The average RMSE and structural similarity (SSIM) index of all slices in the FBP and U-Net reconstructions without and with PWLS for the test ellipsoid phantom are displayed in Table 1. The U-Net reduces the average RMSE value from $2.55 \times 10^{-3} \mu \mathrm{m}^{-1}$ in $\boldsymbol{f}_{\mathrm{FBP}}$ to $1.21 \times 10^{-3} \mu \mathrm{m}^{-1}$ in $\boldsymbol{f}_{\mathrm{U}-\mathrm{Net}}$. With PWLS, the average RMSE is further reduced to $1.16 \times 10^{-3} \mu \mathrm{m}^{-1}$ in $\boldsymbol{f}_{\mathrm{U}-\mathrm{Net} \text {, PWLS }}$. Consistently, $\boldsymbol{f}_{\mathrm{U}-\mathrm{Net}, \mathrm{PWLS}}$ achieves a larger SSIM index than $\boldsymbol{f}_{\mathrm{U}-\mathrm{Net}}$. This quantitative evaluation also demonstrates the efficacy of the U-Net in limited angle tomography and the benefit of PWLS preprocessing.

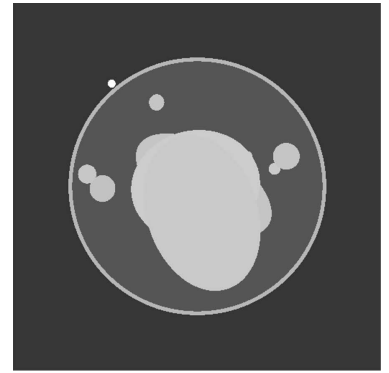

(a)

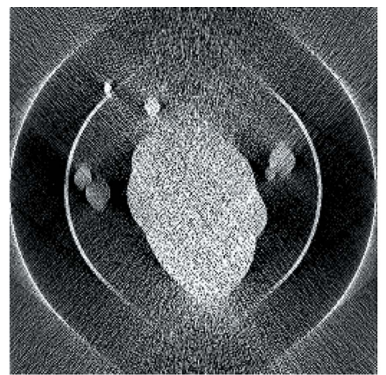

(b)

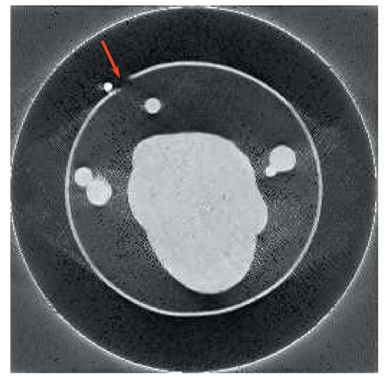

(d)

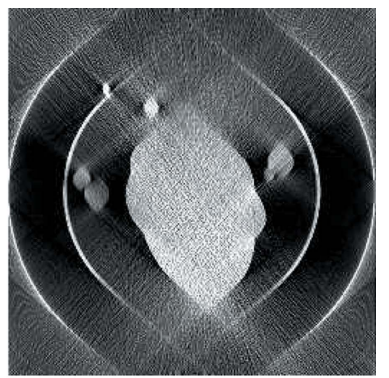

(c)

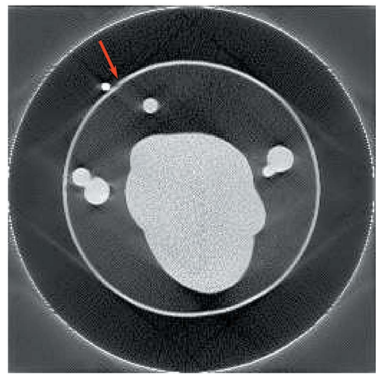

(e)
The reconstruction results of the 215th slice in the test ellipsoid phantom without and with PWLS using a photon number of $10^{4}$, window $[0,0.02] \mu^{-1}$. The boundary indicated by the red arrow is disconnected in $\boldsymbol{f}_{\mathrm{U}-\mathrm{Net}}$, while it is reconstructed in $\boldsymbol{f}_{\mathrm{U}-\mathrm{Net}, \mathrm{PWLS}}$. The RMSE of each image with respect to the corresponding reference with the unit $10^{-3} \mu \mathrm{m}^{-1}$ is as follows: (a) $\boldsymbol{f}_{\text {Reference }} ;(b) \boldsymbol{f}_{\mathrm{FBP}}, 3.61$; (c) $\boldsymbol{f}_{\mathrm{FBP}, \mathrm{PWLS}}, 3.45 ;(d) \boldsymbol{f}_{\mathrm{U}-\mathrm{Net}}, 1.65$; and $(e) \boldsymbol{f}_{\mathrm{U}-\mathrm{Net}, \mathrm{PWLS}}, 1.58$. 


\subsection{Chlorella results}

To demonstrate the benefit of PWLS for the chlorella data, horizontal slices are reconstructed by FBP from the chlorella projection data without or with PWLS processing. A 3D volume is obtained by stacking the horizontal slices. Sagittal slices are obtained by reslicing the volume into 256 slices in the sagittal view. The sagittal slices from projections without and with PWLS are denoted by $\boldsymbol{f}_{\text {sag, FBP }}$ and $\boldsymbol{f}_{\text {sag, FBP, PWLS, }}$ respectively. The results of the 103rd slice are displayed in Fig. 5. Fig. 5(a) shows that the subcellular structures of cell wall, chloroplast, lipid bodies, nucleus and pyrenoid are reconstructed. However, because of noise, the nucleus membrane is barely seen, which is indicated by the red solid

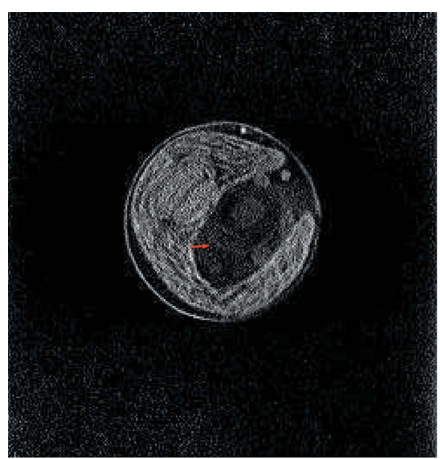

(a)

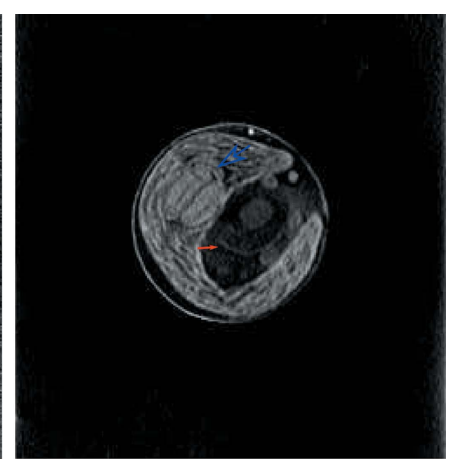

(b)

Figure 5

The 103rd slice from the sagittal view reconstructed from projections without and with PWLS pre-processing. The nucleus membrane in $(a)$ and $(b)$ is indicated by the red solid arrow. The pyrenoid membrane is indicated by the blue hollow arrow. Window: $[0,0.015] \mu \mathrm{m}^{-1}$. (a) $f_{\mathrm{sag}, \text { FBP }}$ and $(b) \boldsymbol{f}_{\text {sag, FBP, PWLS }}$.

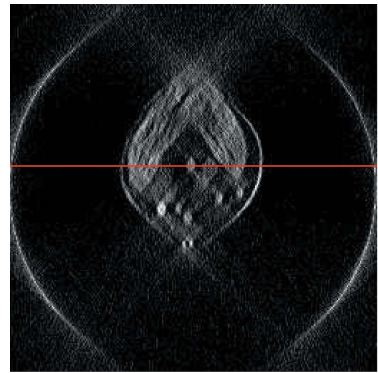

(a)

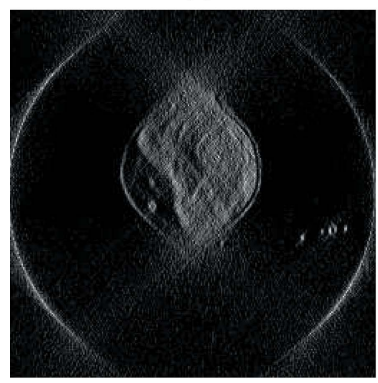

(e)

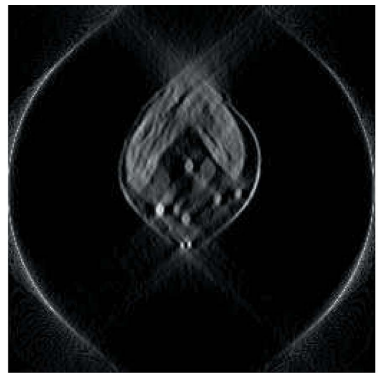

(b)

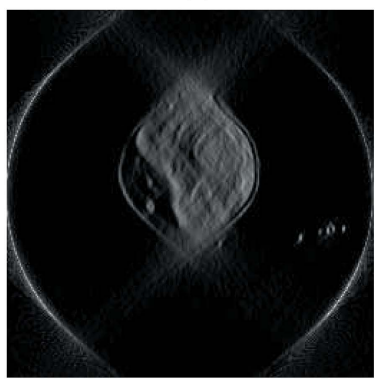

(f)

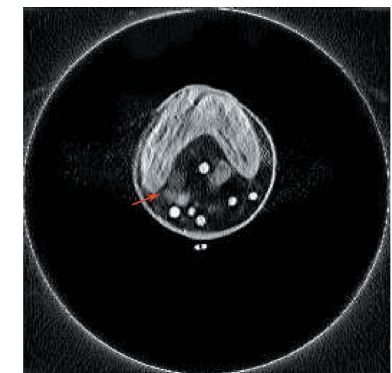

(c)

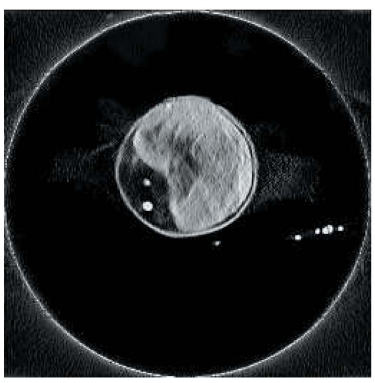

(g)

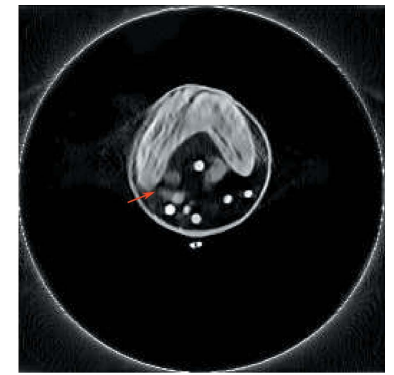

(d)

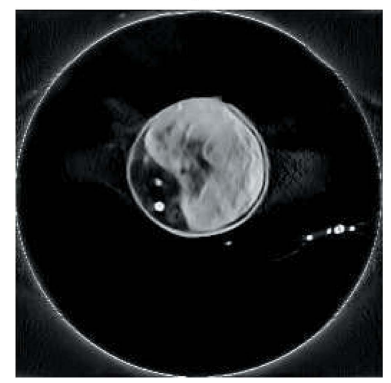

(h)

Figure 6

The reconstruction results of two horizontal slices for the chlorella data, window: $[0.003,0.015] \mu \mathrm{m}^{-1}$. The top row is for the 213 th slice while the bottom row is for the $331 \mathrm{st}$ slice. The red line in $(a)$ indicates the position of intensity profiles in Fig. 7. $(a) \boldsymbol{f}_{\mathrm{FBP}},(b) \boldsymbol{f}_{\mathrm{FBP}, \mathrm{PWLS}},(c) \boldsymbol{f}_{\mathrm{U}-\mathrm{Net}},(d) \boldsymbol{f}_{\mathrm{U}-\mathrm{Net}}$ PWLS,$(e) \boldsymbol{f}_{\mathrm{FBP}}$, $(f) \boldsymbol{f}_{\mathrm{FBP}, \mathrm{PWLS}},(g) \boldsymbol{f}_{\mathrm{U}-\mathrm{Net}}$ and $(h) \boldsymbol{f}_{\mathrm{U}-\mathrm{Net}, \mathrm{PWLS}}$. 


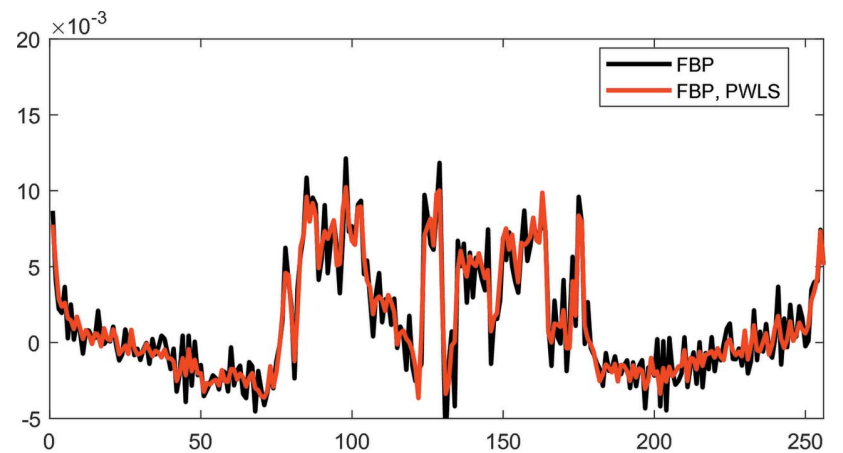

(a)

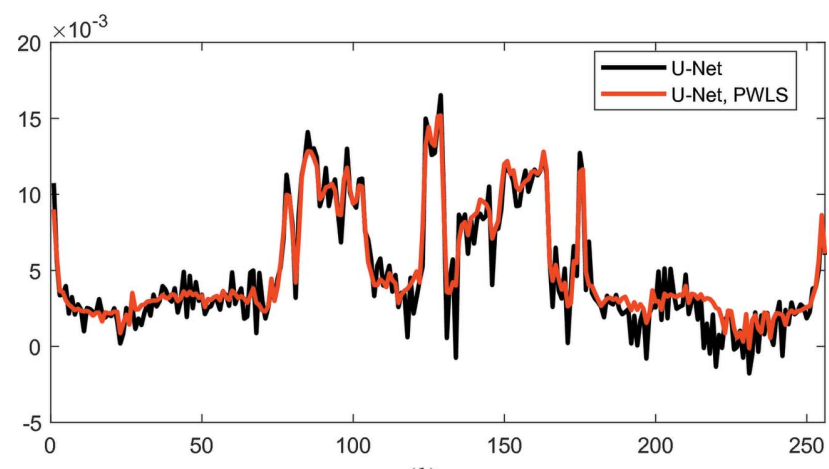

(b)

Figure 7

The intensity profiles of a line in the FBP and U-Net reconstructions without and with PWLS. The position of the line is indicated in Fig. 6(a). (a) Line profiles of FBP reconstructions and (b) line profiles of U-Net reconstructions.

PWLS are displayed in Fig. 7. The position of the line is indicated in Fig. 6(a). In Fig. 7( $a)$, the line profiles of $\boldsymbol{f}_{\mathrm{FBP}}$ and $\boldsymbol{f}_{\mathrm{FBP}}$ PWLS are displayed. For both profiles, in the pixels of 0-70 and 180-256, the intensity value increases from the center outward, which is a characteristic of cupping artifacts and indicates the existence of data truncation. In the profile of $f_{\text {FBP }}$, a lot of high-frequency oscillations are observed, while many of them are mitigated in $\boldsymbol{f}_{\mathrm{FBP} \text {, PWLS }}$ by PWLS. In Fig. 7(b), high frequency oscillations are observed in the profile of $\boldsymbol{f}_{\mathrm{U}-\mathrm{Net}}$ as well, while the profile of $\boldsymbol{f}_{\mathrm{U}-\mathrm{Net} \text {, PWLS }}$ has relatively smooth transitions. This demonstrates the benefit of PWLS in avoiding high-frequency noise in the U-Net reconstruction.

In the sagittal view, although structures are observed well for central slices such as the 103rd slice, structures in many other slices are distorted because of missing data. For example, the 150th sagittal slice of the FBP reconstruction $\boldsymbol{f}_{\mathrm{FBP}, \text { PWLS }}$ is displayed in Fig. $8(a)$, where the cell wall is severely distorted. With the proposed U-Net reconstruction with PWLS pre-processing, the cell wall is restored in an approximate round shape, as shown in Fig. 8(b).

The volumes reconstructed by FBP and U-Net with PWLS are rendered by ParaView, an open-source $3 \mathrm{D}$ visualization tool, and displayed in Figs. 9(a) and 9(b), respectively. Fig. 9(a) shows that the top and bottom parts of the chlorella cell are missing. In addition, the shapes of lipid bodies are distorted. Instead, the top and bottom parts are restored by the U-Net and the lipid body shapes are also restored to round shapes.

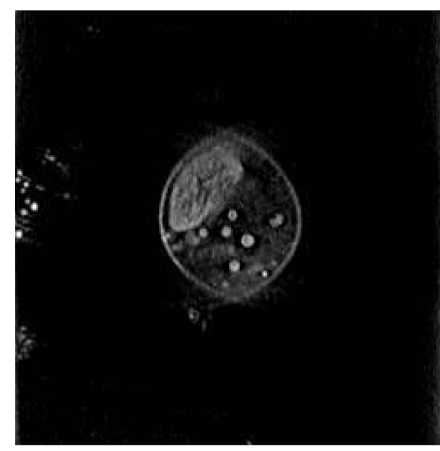

(a)

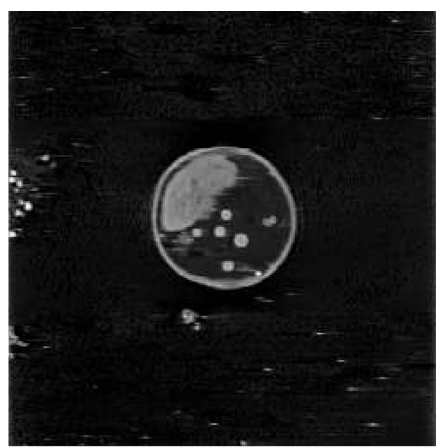

(b)
Figure 8

The 150th slices from the sagittal view reconstructed by FBP and U-Net with PWLS pre-processing. Window: $[0,0.015] \mu \mathrm{m}^{-1}$. (a) $\boldsymbol{f}_{\text {sag, PwLS }}$ and (b) $f_{\text {sag, U-Net, PWLS }}$.

Moreover, in the U-Net reconstruction, the lipid bodies indicated by the arrows are observed well while they are barely seen in the FBP reconstruction. This 3D rendering result highlights the benefit of U-Net in the 3D visualization of subcellular structures.

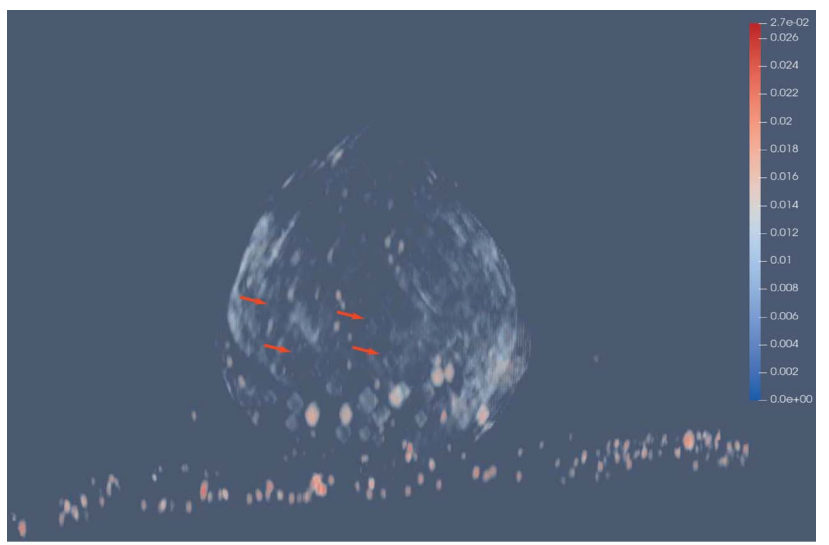

(a)

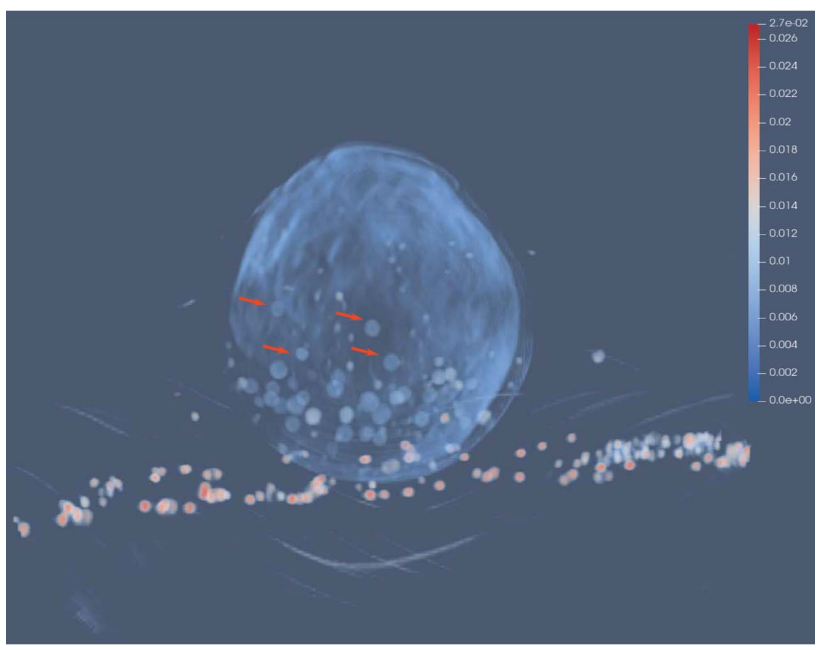

Figure 9

(b)

The 3D rendering of the volumes reconstructed by FBP and U-Net with PWLS using the tool of ParaView, which is viewed along the $z$ direction. The lipids indicated by the arrows are observed well in $f_{\mathrm{U}-\mathrm{Net}}$ PWLS while they are barely seen in $\boldsymbol{f}_{\mathrm{FBP}, \text { PWLS }} \cdot(a) \boldsymbol{f}_{\mathrm{FBP} \text {, PWLS }}$ and $(b) \boldsymbol{f}_{\mathrm{U} \text {-Net, PWLS }}$. 


\subsection{Discussion}

As a state-of-the-art method, the U-Net achieves significant improvement in image quality from the FBP reconstructions, achieving the best average RMSE value in Table 1. However, in some cases, the structures it predicts are not accurate. For example, the cell wall is not in a perfect round shape in Figs. $6(d)$ and $8(b)$. This is potentially caused by various factors such as noise, insufficient training data and over-fitting, which are ineluctable for deep learning. Because of the coexistence of the limited-angle problem and data-truncation problem in this work, where truncation is caused by the largescale ice for immobilization of samples, applying iterative reconstruction such as simultaneous algebraic reconstruction technique with TV regularization for data consistent reconstruction (Huang et al., 2019b) to improve such incorrect structures is not feasible.

In limited angle tomography, only structures whose orientations are tangent to available X-rays can be reconstructed (Quinto, 1993, 2006, 2007; Huang et al., 2016a). Therefore, in the FBP reconstructions, most edges whose orientations are inside the scanned angular range are reconstructed. Because of this, for the chlorella reconstruction, several slices in the sagittal view contain good resolution structures. On the other hand, with the geometry setting in this work, the sagittal slices are equivalent to focus planes in tomosynthesis (Grant, 1972) where most X-rays focus. Therefore, structures viewed in sagittal planes preserve better resolution than any horizontal planes. However, structures are preserved well only in a limited number of central slices in the sagittal view, while most structures are still distorted because of missing data [Fig. 8(a)]. In order to view structures in any intersectional planes, artifact reduction is necessary.

Due to missing data, many essential subcellular structures are distorted or even missing in the FBP reconstruction, e.g. the lipid bodies in this work. The distribution and states of subcellular structures provide crucial information of intracellular activities (Ortega et al., 2009; Wang et al., 2015a). With the power of deep learning in image processing, the proposed reconstruction method is competent for $3 \mathrm{D}$ visualization of subcellular structures, as displayed in Fig. 9. This observation indicates its important value for nanoscale imaging in biology, nanoscience and materials science.

\section{Conclusions and outlook}

In this work, deep learning has been applied to limited angle reconstruction in TXMs for the first time. PWLS pre-processing is beneficial to improving the image quality of deeplearning reconstruction. Despite the limitation to accessing sufficient real training data, this work demonstrates that training a deep neural network model from synthetic data with proper noise modeling is a promising approach. The proposed deep-learning reconstruction method remarkably improves the $3 \mathrm{D}$ visualization of subcellular structures, indicating its important value for nanoscale imaging in biology, nanoscience and materials science.
Although promising and intriguing results are achieved in this work, the limited angle reconstruction problem is still not entirely resolved, since some structures are reconstructed inaccurately. In the future, the following aspects of work are worth investigating. (i) Evaluate the proposed deep-learning reconstruction method on more complex samples. (ii) More realistic noise modeling for synthetic data should potentially improve deep-learning performance. (iii) Explore new approaches to achieve data consistent reconstruction (Huang et al., 2019b) in the co-existence of the limited-angle problem and data-truncation problem. (iv) If possible, building up a database from complete real scans for training deep neural networks is necessary.

\section{Funding information}

We are very grateful for the chlorella data provided by the soft X-ray microscope at beamline BL07W in the National Synchrotron Radiation Laboratory in Hefei, China. The research leading to these results has received funding from the European Research Council (ERC) under the European Union's Horizon 2020 research and innovation programme (ERC grant No. 810316).

\section{References}

Andrews, J. C., Meirer, F., Liu, Y., Mester, Z. \& Pianetta, P. (2011). Microsc. Res. Tech. 74, 671-681.

Barnard, D. P., Turner, J. N., Frank, J. \& McEwen, B. F. (1992). J. Microsc. 167, 39-48.

Baudelet, P.-H., Ricochon, G., Linder, M. \& Muniglia, L. (2017). Algal Res. 25, 333-371.

Brisard, S., Chae, R. S., Bihannic, I., Michot, L., Guttmann, P., Thieme, J., Schneider, G., Monteiro, P. J. \& Levitz, P. (2012). Am. Mineral. 97, 480-483.

Bubba, T. A., Kutyniok, G., Lassas, M., März, M., Samek, W., Siltanen, S. \& Srinivasan, V. (2019). Inverse Probl. 35, 064002.

Chao, W., Harteneck, B. D., Liddle, J. A., Anderson, E. H. \& Attwood, D. T. (2005). Nature, 435, 1210-1213.

Chen, Z., Jin, X., Li, L. \& Wang, G. (2013). Phys. Med. Biol. 58, 2119-2141.

Davison, M. E. (1983). SIAM J. Appl. Math. 43, 428-448.

Defrise, M. \& de Mol, C. (1983). Opt. Acta: Int. J. Opt. 30, 403-408.

Falk, T., Mai, D., Bensch, R., Çiçek, Ö., Abdulkadir, A., Marrakchi, Y., Böhm, A., Deubner, J., Jäckel, Z., Seiwald, K., Dovzhenko, A., Tietz, O., Dal Bosco, C., Walsh, S., Saltukoglu, D., Tay, T. L., Prinz, M., Palme, K., Simons, M., Diester, I., Brox, T. \& Ronneberger, O. (2019). Nat. Methods, 16, 67-70.

Gerchberg, R. (1974). J. Mod. Opt. 21, 709-720.

Grant, D. G. (1972). IEEE Trans. Biomed. Eng. 19, 20-28.

$\mathrm{Gu}$, J. \& Ye, J. C. (2017). Proceedings of the 2017 International Conference on Fully Three-Dimensional Image Reconstruction in Radiology and Nuclear Medicine (Fully3D2017), 18-23 June 2017, Xi'an Shaanxi, China, pp. 443-447.

Helgason, S. (1965). Acta Math. 113, 153-180.

Holler, M., Raabe, J., Wepf, R., Shahmoradian, S. H., Diaz, A., Sarafimov, B., Lachat, T., Walther, H. \& Vitins, M. (2017). Rev. Sci. Instrum. 88, 113701.

Hu, J., Shen, L. \& Sun, G. (2018). Proceedings of the 2018 IEEE Conference on Computer Vision and Pattern Recognition (CVPR), 18-22 June 2018, Salt Lake City, USA, pp. 7132-7141.

Huang, Y., Huang, X., Taubmann, O., Xia, Y., Haase, V., Hornegger, J., Lauritsch, G. \& Maier, A. (2017). Biomed. Phys. Eng. Expr. 3, 035015 . 
Huang, Y., Lauritsch, G., Amrehn, M., Taubmann, O., Haase, V., Stromer, D., Huang, X. \& Maier, A. (2016a). Proceedings of Bildverarbeitung für die Medizin 2016 (BVM2016), 13-15 March 2016, Berlin, Germany, pp. 277-282. Springer.

Huang, Y., Lu, Y., Taubmann, O., Lauritsch, G. \& Maier, A. (2019a). Int. J. Comput. Assist. Radiol. Surg. 14, 11-19.

Huang, Y., Preuhs, A., Lauritsch, G., Manhart, M., Huang, X. \& Maier, A. (2019b). arXiv:1908.06792.

Huang, Y., Taubmann, O., Huang, X., Haase, V., Lauritsch, G. \& Maier, A. (2016b). IEEE 13th International Symposium on Biomedical Imaging (ISBI), 13-16 April 2016, Prague, Czech Republic, pp. 585-588. IEEE.

Huang, Y., Taubmann, O., Huang, X., Haase, V., Lauritsch, G. \& Maier, A. (2018a). IEEE Trans. Radiat. Plasma Med. Sci. 2, 307-314.

Huang, Y., Taubmann, O., Huang, X., Lauritsch, G. \& Maier, A. (2018b). Proceedings of CT Meeting, pp. 189-192.

Huang, Y., Würfl, T., Breininger, K., Liu, L., Lauritsch, G. \& Maier, A. (2018c). Proceedings of the 21st International Conference on Medical Image Computing and Computer-Assisted Intervention (MICCAI2018), 16-20 September 2018, Granada, Spain, pp. 145-153. Cham: Springer International Publishing.

Ioffe, S. \& Szegedy, C. (2015). arXiv:1502.03167.

Koster, A. J., Grimm, R., Typke, D., Hegerl, R., Stoschek, A., Walz, J. \& Baumeister, W. (1997). J. Struct. Biol. 120, 276-308.

Kudo, H. \& Saito, T. (1991). J. Opt. Soc. Am. A, 8, 1148-1160.

Li, F.-F., Fergus, R. \& Perona, P. (2006). IEEE Trans. Pattern Anal. Mach. Intell. 28, 594-611.

Liu, J., Li, F., Chen, L., Guan, Y., Tian, L., Xiong, Y., Liu, G. \& Tian, Y. (2018). J. Microsc. 270, 64-70.

Louis, A. K. (1981). Mathematical Aspects of Computerized Tomography, edited by G. T. Herman \& F. Natterer, pp. 127-139. Berlin: Springer.

Louis, A. K. (1986). Numer. Math. 48, 251-262.

Louis, A. K. \& Törnig, W. (1980). Math. Methods Appl. Sci. 2, 209-220.

Ludwig, D. (1966). Commun. Pure Appl. Math. 19, 49-81.

Maier, A. K., Syben, C., Stimpel, B., Würfl, T., Hoffmann, M., Schebesch, F., Fu, W., Mill, L., Kling, L. \& Christiansen, S. (2019). Nat. Mach. Intell. 1, 373-380.

Natterer, F. (1986). The Mathematics of Computerized Tomography. Chichester: John Wiley \& Sons.

Nelson, J., Misra, S., Yang, Y., Jackson, A., Liu, Y., Wang, H., Dai, H., Andrews, J. C., Cui, Y. \& Toney, M. F. (2012). J. Am. Chem. Soc. 134, 6337-6343.

Odena, A., Dumoulin, V. \& Olah, C. (2016). Distill, 1, e3.

Ortega, R., Deves, G. \& Carmona, A. (2009). J. R. Soc. Interface, 6(Suppl. 5), S649-S658.

Palatucci, M., Pomerleau, D., Hinton, G. E. \& Mitchell, T. M. (2009). Proceedings of Neural Information Processing Systems (NIPS), Vol. 22, pp. 1410-1418.

Pan, S. J. \& Yang, Q. (2010). IEEE Trans. Knowl. Data Eng. 22, 1345-1359.

Papoulis, A. (1975). IEEE Trans. Circuits Syst. 22, 735-742.
Prince, J. L. \& Willsky, A. S. (1990). Opt. Eng. 29, 535-544.

Qu, G. R. \& Jiang, M. (2009). Acta Math. Appl. Sin. Engl. Ser. 25, 327-334.

Qu, G. R., Lan, Y. S. \& Jiang, M. (2008). Acta Math. Appl. Sin. Engl. Ser. 24, 157-166.

Quinto, E. T. (1993). SIAM J. Math. Anal. 24, 1215-1225.

Quinto, E. T. (2006). The Radon Transform, Inverse Problems, and Tomography, Volume 63 of Proceedings of Symposia in Applied Mathematics, pp. 1-24. American Mathematical Society.

Quinto, E. T. (2007). J. Comput. Appl. Math. 199, 141-148.

Ritschl, L., Bergner, F., Fleischmann, C. \& Kachelriess, M. (2011). Phys. Med. Biol. 56, 1545-1561.

Ronneberger, O., Fischer, P. \& Brox, T. (2015). Proceedings of the 18th International Conference on Medical Image Computing and Computer-Assisted Intervention (MICCAI20), Munich, Germany, pp. 234-241. Springer.

Sakdinawat, A. \& Attwood, D. (2010). Nat. Photon. 4, 840-848.

Shapiro, D., Thibault, P., Beetz, T., Elser, V., Howells, M., Jacobsen, C., Kirz, J., Lima, E., Miao, H., Neiman, A. M. \& Sayre, D. (2005). Proc. Natl Acad. Sci. 102, 15343-15346.

Shearing, P., Bradley, R., Gelb, J., Lee, S., Atkinson, A., Withers, P. \& Brandon, N. (2011). Electrochem. Solid-State Lett. 14, B117-B120.

Sidky, E. Y., Kao, C.-M. \& Pan, X. (2006). J. X-ray Sci. Technol. 14, 119-139.

Sidky, E. Y. \& Pan, X. (2008). Phys. Med. Biol. 53, 4777-4807.

Smit, E. de, Swart, I., Creemer, J. F., Hoveling, G. H., Gilles, M. K., Tyliszczak, T., Kooyman, P. J., Zandbergen, H. W., Morin, C., Weckhuysen, B. M. \& de Groot, F. M. F. (2008). Nature, 456 , 222-225.

Wang, J., Li, T., Lu, H. \& Liang, Z. (2006). IEEE Trans. Med. Imaging, 25, 1272-1283.

Wang, L., Zhang, T., Li, P., Huang, W., Tang, J., Wang, P., Liu, J., Yuan, Q., Bai, R., Li, B., Zhang, K., Zhao, Y. \& Chen, C. (2015a). ACS Nano, 9, 6532-6547.

Wang, P., Lombi, E., Zhao, F.-J. \& Kopittke, P. M. (2016). Trends Plant Sci. 21, 699-712.

Wang, S., Liu, J., Li, Y., Chen, J., Guan, Y. \& Zhu, L. (2019). J. Synchrotron Rad. 26, 1808-1814.

Wang, S., Wang, D., Wu, Q., Gao, K., Wang, Z. \& Wu, Z. (2015b). J. Synchrotron Rad. 22, 1091-1095.

Wang, Y., Jacobsen, C., Maser, J. \& Osanna, A. (2000). J. Microsc. 197, 80-93.

Würfl, T., Ghesu, F. C., Christlein, V. \& Maier, A. (2016). Proceedings of the 19th International Conference on Medical Image Computing and Computer-Assisted Intervention (MICCAI2016), 17-21 October 2016, Athens, Greece, pp. 432-440. Springer.

Würfl, T., Hoffmann, M., Christlein, V., Breininger, K., Huang, Y., Unberath, M. \& Maier, A. K. (2018). IEEE Trans. Med. Imaging, 37, 1454-1463.

Yang, Y., Yang, F., Hingerl, F. F., Xiao, X., Liu, Y., Wu, Z., Benson, S. M., Toney, M. F., Andrews, J. C. \& Pianetta, P. (2015). J. Synchrotron Rad. 22, 452-457.

Yu, H., Xia, S., Wei, C., Mao, Y., Larsson, D., Xiao, X., Pianetta, P., Yu, Y.-S. \& Liu, Y. (2018). J. Synchrotron Rad. 25, 1819-1826. 